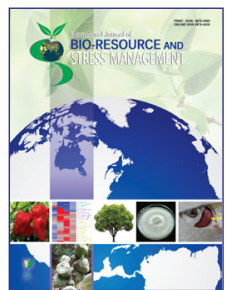

\title{
Walking and Dry Season Stresses on Crossbred Cows of Andaman and Nicobar Islands
}

\author{
P. Perumal*, A. K. De, D. Bhattacharya, A. Kundu, Jai Sunder, K. Muniswamy, S. K. Ravi and Sneha Bhowmick
}

ICAR-Central Island Agricultural Research Institute, Port Blair, Andaman and Nicobar Islands (744 101), India

Open Access

\section{Corresponding Author}

P. Perumal

e-mail: perumalponraj@gmail.com

Citation: Perumal et al., 2019. Walking and dry season stresses on crossbred cows of Andaman and Nicobar Islands. International Journal of Bio-resource and Stress Management 2019, 10(5):520-528. HTTPS:// DOI.ORG/10.23910/IJBSM/2019.10.5.2027a

Copyright: (c) 2019 Perumal et al. This is an open access article that permits unrestricted use, distribution and reproduction in any medium after the author(s) and source are credited.

Data Availability Statement: Legal restrictions are imposed on the public sharing of raw data. However, authors have full right to transfer or share the data in raw form upon request subject to either meeting the conditions of the original consents and the original research study. Further, access of data needs to meet whether the user complies with the ethical and legal obligations as data controllers to allow for secondary use of the data outside of the original study.

Funding: The research was conducted with the kind and supports from Institute, ICAR-Central Island Agricultural Research Institute, Port Blair, Andaman and Nicobar Islands.

Conflict of interests: The authors have declared that no conflict of interest exists.

Acknowledgement: Authors acknowledge the supports from the Director, ICAR-Central Island Agricultural Research Institute, Port Blair, Andaman and Nicobar Islands and Head of the Animal Science Division of the institute.

\begin{abstract}
An investigation was carried out to determine the walking stress effect during dry season on physiological, biochemical and heamatological profiles in crossbred cows of Andaman and Nicobar Islands (ANI), India. Twelve numbers of adult crossbred cows of 4-6 yr of age with good body condition (score 5-6) were selected for the experiment. They were categorised into two groups; Group I: control (not exposed to walking stress effect) and Group II: treatment (exposed to walking stress effect) and each group consisted of six animals. In the stress induced group, the cows were walked to $10 \mathrm{~km}$ to and fro in the undulated area or hilly areas without allowing grazing from the cattle farm. While control group animals were protected in the separate nearby cattle shed roofed with asbestos sheets with surrounded big trees. The cows walked from 0700 to $1200 \mathrm{~h}$ during dry season, i.e. November to February and restrained properly and routine physiological parameters were scaled immediately return from stressful walk during dry season. Similarly blood was collected simultaneously and assessed the routine common heamatological profiles. Antioxidant profiles and lipid peroxide profile were also measured in treatment and control animal groups. Data analysis revealed that physiological, heamatological profiles and also MDA concentration were increased significantly $(p<0.05)$ and concentration of antioxidants were decreased significantly $(p<0.05)$ in the treatment crossbred cows than in control animal group. The study concludes that walking stress during dry season has significantly $(p<0.05)$ influenced the performance of crossbred cows of ANI.
\end{abstract}

Keywords: Cows, walking, stress, season, physiological, heamatological, antioxidant profiles

\section{Introduction}

Reproduction and production performances differ with various factors and these factors ultimately decide the profit of the dairy herd. According to data availability in the Agriculture Ministry, Govt. of India, milk production was 15000 t from 52571 cattle heads with per capita availability of milk was $110 \mathrm{~g} \mathrm{day}^{-1}$ in Andaman and Nicobar Islands whereas in India national average was $337 \mathrm{~g}$ (Livestock Census of India, 2012). According to the Vision 2050 of ICAR-CIARI, the milk production deficit was $44 \%$ in 2011 and it will increase more in 2050 in the A \& N Islands. Per capita availability and milk production were decreased over a period of the time from 2001 to 2016 in the A \& N Islands. According to the livestock censuses of Govt. of India (2003; 2007; 2012), the cattle population is decreased due to various reasons. Therefore, it is high time that to get sincere efforts from the different sectors like veterinary \& animal husbandry

\section{Article History}

RECEIVED in $24^{\text {th }}$ August 2019 RECEIVED in revised form 24 $4^{\text {th }}$ October 2019 ACCEPTED in final form $29^{\text {th }}$ October 2019 
department, policy makers, cattle breeders and farmers, cattle researchers to work out on how to increase the crossbred cattle efficiency through ideal and optimum management to improve the production and reproductive performances to increase the socio-economic status of the dairy farmers of ANI. Crossbred cows are reared through semi-intensive system of rearing and in the system; the animals need to be walked for longer distance to receive sufficient feed/fodder in island ecosystem of ANI mainly during dry season. Green fodder or grasses availability for grazing in the grassing area is often of poor quality with low densities per unit area and also the time for grazing is limited only (day time) with exposure to solar radiation and fly nuisance and insect irritation (Manteca and Smith, 1994). Strenuous walking induces not only stress to the dairy cows, but also loss of energy leads to reduced production capacity and reproductive failure, ultimately profit loss in the dairy sector. Different stresses causes losses are occurred more prominently and significantly during dry or summer season and also observed that minimization of the walking distance for exercise or grazing, the productive as well as the reproductive performance of the dairy cows has been improved significantly (Gustafson et al., 1993) and breed variation was also determined the susceptibility of animals to various stresses in the livestock species (D'Hour et al., 1994). Perusal of literature availability, it was observed that there was no information on walking and dry season stress in crossbred cows of ANI. Therefore, a study was carried out to estimate the walking stress effect during dry season on heamatological, physiological and antioxidant profiles in crossbred cows of ANI under tropical island ecosystem.

\section{Materials and Methods}

\subsection{Location}

Present study was conducted at livestock farm, ICAR-CIARI, Port Blair, ANI and is placed in between 6⒋ $45^{\prime}$ to $13^{\circ} 41^{\prime}$ North Latitude and in between $92^{\circ} 12^{\prime}$ to $93^{\circ} 57^{\prime}$ East Longitude. Average maximum and minimum temperature were 30.1 and $23^{\circ} \mathrm{C}$, respectively. Relative humidity was in range of $82-94 \%$ and annual rainfall is $>3100 \mathrm{~mm}$ spread over $>8$ months in a year is called as monsoon season whereas remaining from November to February is called as dry season means very less rainfall. The present study was conducted during dry season. Crossbred cows of ANI were maintained in the semiintensive system where they were allowed for grazing from 0700 to $1200 \mathrm{~h}$. Feeding and watering were done as per the farm schedule.

\subsection{Experimental animals}

Twelve apparently healthy adult crossbred cows of 4 - 6 yr of age with good body condition (score 5-6) were selected from the cattle herd in livestock farm. They were maintained under hygienic managemental conditions. Prophylactic measures like deworming and vaccination were done as per farm schedule.

\subsection{Housing management}

Animal shed was prepared with asbestos-roof and half of the wall made up of wire mesh and half was by bricks wall in all the four side of the shed with surrounding of the big trees. Experimental cows were placed under homogeneous feeding, watering, lighting and other managemental conditions. At $0700 \mathrm{~h}$, both the groups of experiment were released from the shed. At $1200 \mathrm{~h}$, all the cows were properly restrained in their original positions in the shed. This whole procedure was followed throughout the investigation period.

\subsection{Experimental procedure}

The study was carried out for a period of 7 weeks during dry season. These cows were selected randomly into two groups and each group consisted of six animals: Group I (control) and Group II (treatment: walking stress). Both groups were stallfed and watered at $1200 \mathrm{~h}$ and allowed access to ad libitum water throughout the day. Walking stress group (Group II) cows were made to walk for $10 \mathrm{~km}$. Blood samples were collected at week interval to assess the walking stress effect on the heamatological and antioxidant profiles.

\subsection{Blood collection and plasma separation}

Five $\mathrm{ml}$ of blood was collected from both the groups from external jugular vein in tubes containing heparin $\left(20 \mathrm{IU} / \mathrm{ml}^{-1}\right.$ of blood) at $1200 \mathrm{~h}$ immediately after completing the walking. Complete blood profiles were estimated and plasma was separated by centrifugation at $3500 \times \mathrm{g}$ at $4{ }^{\circ} \mathrm{C}$ for $10 \mathrm{~min}$. Separated plasma was aliquoted into small aliquots in microcryo-tubes and placed frozen at $-20{ }^{\circ} \mathrm{C}$ until further analysis of biochemical and antioxidant profiles with commercial diagnostic kits.

\subsection{Parameters studied}

Physiological profiles such as rectal temperature (RT), respiration rate (RR), pulse rate (PR), heart rate $(H R)$ and skin temperature (ST) were recorded twice daily at 0700 and $1200 \mathrm{~h}$. Blood profiles such as red blood cells (RBC), haemoglobin $(\mathrm{Hb})$, erythrocyte sedimentation rate (ESR), packed cell volume (PCV), mean corpuscular volume (MCV), mean corpuscular haemoglobin (MCH), and mean corpuscular haemoglobin concentration ( $\mathrm{MCHC}$ ) were estimated by automatic blood analyser. Antioxidant profiles such as like superoxide dismutase (SOD), reduced glutathione (GSH), catalase (CAT) and total antioxidant capacity (TAC) and lipid peroxide profile (malondialdehyde; MDA) were estimated using commercial diagnostic kits.

\subsection{Statistical analysis}

The data was analysed statistically and expressed as the mean \pm S.E.M. Means were analyzed by one way analysis of variance followed by Tukey's post hoc test to determine significant differences among the weeks and student " $t$ " test was for between the treatment and control groups using the SPSS/PC computer program (version 15.0; SPSS, Chicago, IL). Differences with values of $p<0.05$ were considered to be 
statistically significant.

\section{Results and Discussion}

Physiological, heamatological and antioxidant profiles were significantly altered due to walking stress in crossbred cows causes the reproductive and production performance were adversely affected in stressed cows. Thus it shows a clear picture that walking or exercising or grazing cows requires higher balanced nutrition with higher energy to counteract or overcome the deleterious effects of stress caused by walking and measurement of such effect of walking stress in cows will understand to the cattle growers, dairy farmers and cattle breeders to know and to acclimatise suitable protecting methodologies with or without nutritional influence to avoid energy wastage in the exercise or walking. In the current study particularly to understand that the walking effect during dry season on reproductive and production performances assists to maintain the crossbred cows more effectively and efficiently in ANI under tropical humid island ecosystem.

Walking stress in dry season on physiological (Table 1) and heamatological (Table 2) attributes for different seven weeks between the treatment and control groups were depicted

Table 1: Mean ( \pm S.E.) physiological parameters of crossbred cows of Andaman and Nicobar Islands exposed to walking stress at different weeks

\begin{tabular}{|c|c|c|c|c|c|c|c|c|c|}
\hline \multirow{2}{*}{\multicolumn{2}{|c|}{$\begin{array}{l}\text { Param- } \\
\text { eters }\end{array}$}} & \multicolumn{7}{|c|}{ Experimental periods } & \multirow[t]{2}{*}{ Overall } \\
\hline & & \multirow{2}{*}{$\begin{array}{c}1^{\text {st }} \text { week } \\
99.73 \pm\end{array}$} & \multirow{2}{*}{$\begin{array}{c}2^{\text {nd }} \text { week } \\
100.14 \pm\end{array}$} & \multirow{2}{*}{$\frac{3^{\text {rd }} \text { week }}{99.87 \pm}$} & \multirow{2}{*}{$\frac{4^{\text {th }} \text { week }}{99.43 \pm}$} & \multirow{2}{*}{$\begin{array}{c}5^{\text {th }} \text { week } \\
99.54 \pm\end{array}$} & \multirow{2}{*}{$\frac{6^{\text {th }} \text { week }}{100.87 \pm}$} & \multirow{2}{*}{$\begin{array}{c}7^{\text {th }} \text { week } \\
100.32 \pm\end{array}$} & \\
\hline RT & GI & & & & & & & & $99.98 \pm$ \\
\hline & & $1.12^{\mathrm{aA}}$ & $1.19^{\mathrm{abA}}$ & $1.26^{\mathrm{aA}}$ & $1.23^{\mathrm{aA}}$ & $1.12^{\mathrm{aA}}$ & $1.14^{\mathrm{bA}}$ & $1.15^{\mathrm{abA}}$ & $0.93^{A}$ \\
\hline & GII & $104.76 \pm$ & $103.56 \pm$ & $103.74 \pm$ & $103.76 \pm$ & $103.34 \pm$ & $104.79 \pm$ & $104.65 \pm$ & $104.16 \pm$ \\
\hline & & $1.32^{\mathrm{cB}}$ & $1.34^{\mathrm{aB}}$ & $1.34^{\mathrm{abB}}$ & $1.28^{\mathrm{abcB}}$ & $1.14^{\mathrm{aB}}$ & $0.98^{\mathrm{cB}}$ & $1.12^{\mathrm{bcB}}$ & $0.98^{\mathrm{B}}$ \\
\hline \multirow[t]{4}{*}{$\mathrm{RR}$} & GI & $27.85 \pm$ & $28.34 \pm$ & $27.78 \pm$ & $27.33 \pm$ & $27.76 \pm$ & $29.08 \pm$ & $28.56 \pm$ & $27.98 \pm$ \\
\hline & & $1.44^{\mathrm{abA}}$ & $1.28^{\mathrm{abA}}$ & $1.21^{\mathrm{abA}}$ & $1.38^{\mathrm{aA}}$ & $1.32^{\mathrm{abA}}$ & $1.51^{\mathrm{bA}}$ & $1.66^{\mathrm{abA}}$ & $0.87^{A}$ \\
\hline & GII & $42.65 \pm$ & $37.65 \pm$ & $39.98 \pm$ & $39.87 \pm$ & $37.82 \pm$ & $43.67 \pm$ & $43.63 \pm$ & $40.72 \pm$ \\
\hline & & $1.43^{\mathrm{cB}}$ & $1.32^{\mathrm{aB}}$ & $1.55^{\mathrm{bB}}$ & $1.74^{\mathrm{bB}}$ & $1.43^{\mathrm{aB}}$ & $1.65^{\mathrm{cB}}$ & $1.56^{\mathrm{cB}}$ & $1.82^{\mathrm{B}}$ \\
\hline \multirow[t]{4}{*}{ PR } & GI & $66.12 \pm$ & $66.43 \pm$ & $66.32 \pm$ & $65.45 \pm$ & $65.97 \pm$ & $67.76 \pm$ & $66.89 \pm$ & $66.45 \pm$ \\
\hline & & $1.24^{\mathrm{abA}}$ & $1.25^{\mathrm{abA}}$ & $1.34^{\mathrm{abA}}$ & $1.37^{\mathrm{aA}}$ & $1.13^{\mathrm{abA}}$ & $1.47^{\mathrm{cA}}$ & $1.34^{\mathrm{bcA}}$ & $0.76^{A}$ \\
\hline & GII & $76.89 \pm$ & $74.23 \pm$ & $75.54 \pm$ & $74.53 \pm$ & $74.53 \pm$ & $78.31 \pm$ & $77.31 \pm$ & $75.91 \pm$ \\
\hline & & $1.19^{\mathrm{bB}}$ & $1.58^{\mathrm{aB}}$ & $1.43^{\mathrm{aB}}$ & $1.18^{\mathrm{aB}}$ & $1.19^{\mathrm{aB}}$ & $1.42^{\mathrm{bB}}$ & $1.43^{\mathrm{bB}}$ & $1.42^{\mathrm{B}}$ \\
\hline \multirow[t]{4}{*}{$\mathrm{HR}$} & GI & $72.31 \pm$ & $72.54 \pm$ & $72.64 \pm$ & $72.65 \pm$ & $72.54 \pm$ & $72.87 \pm$ & $73.42 \pm$ & $72.54 \pm$ \\
\hline & & $1.14^{\mathrm{aA}}$ & $2.28^{\mathrm{abA}}$ & $1.18^{\mathrm{abA}}$ & $1.19^{\mathrm{abA}}$ & $1.28^{\mathrm{abA}}$ & $1.24^{\mathrm{abA}}$ & $1.37^{\mathrm{bA}}$ & $0.87^{A}$ \\
\hline & GII & $82.23 \pm$ & $80.63 \pm$ & $80.52 \pm$ & $80.87 \pm$ & $80.31 \pm$ & $82.76 \pm$ & $83.87 \pm$ & $81.43 \pm$ \\
\hline & & $1.45^{\mathrm{bcB}}$ & $1.43^{\mathrm{aB}}$ & $1.54^{\mathrm{aB}}$ & $1.44^{\mathrm{abB}}$ & $1.52^{\mathrm{aB}}$ & $1.51^{\mathrm{cdB}}$ & $1.32^{\mathrm{dB}}$ & $1.32^{\mathrm{B}}$ \\
\hline \multirow[t]{4}{*}{ ST } & GI & $98.56 \pm$ & $99.33 \pm$ & $98.83 \pm$ & $98.25 \pm$ & $98.76 \pm$ & $99.91 \pm$ & $98.76 \pm$ & $98.86 \pm$ \\
\hline & & $1.21^{\mathrm{aA}}$ & $1.38^{\mathrm{abA}}$ & $1.28^{\mathrm{abA}}$ & $1.33^{\mathrm{aA}}$ & $1.23^{\mathrm{abA}}$ & $1.26^{\mathrm{bA}}$ & $1.23^{\mathrm{abA}}$ & $0.98^{A}$ \\
\hline & GII & $101.81 \pm$ & $101.45 \pm$ & $101.65 \pm$ & $100.47 \pm$ & $100.67 \pm$ & $102.31 \pm$ & $101.55 \pm$ & $101.43 \pm$ \\
\hline & & $1.32^{\mathrm{bcB}}$ & $1.23^{\mathrm{abcB}}$ & $1.31^{\mathrm{abcB}}$ & $1.28^{\mathrm{aB}}$ & $1.32^{\mathrm{abB}}$ & $1.16^{\mathrm{cB}}$ & $1.42^{\mathrm{bcB}}$ & $0.91^{\mathrm{B}}$ \\
\hline
\end{tabular}

RT: Rectal Temperature ( $\left.{ }^{\circ} \mathrm{F}\right) ;$ RR: Respiration Rate (beats per minute); PR: Pulse Rate (beats per minute); HR: Heart Rate (beats per minute); ST: Skin Temperature ( $\left.{ }^{\circ} \mathrm{F}\right)$; GI: Unstressed animal group; GII: Stressed animal group; Within rows means with different letters $(a, b, c, d)$ differ significantly $(p<0.05)$; Within columns means with different letters $(A, B)$ differ significantly $(p<0.05)$ between the stressed and unstressed for the particular parameter

in tables and also coefficients of correlation between the heamatological \& physiological attributes were depicted in treatment (stressed; Table 3) as well as untreated control (unstressed; Table 4) crossbred cows. Through search and analysis of literature, it was revealed there was no information on walking effect during dry season on physiological, haematological and antioxidant profiles for island crossbred cows. Previous authors tested and expressed that the stressful effect due to walking or working during dry season leads to severe adverse consequences on physiological, reproduction and production profiles in various animal species (Daramola and Adeloye, 2009; Sejian et al., 2010b; Perumal et al., 2016).

\subsection{Physiological profiles}

Significant $(p<0.05)$ variation was noticed between the treatment and control groups in various weeks of the 
Table 2: Mean ( \pm S.E.) heamatological parameters of crossbred cows of Andaman and Nicobar Islands exposed to walking stress at different weeks

\begin{tabular}{|c|c|c|c|c|c|c|c|c|c|}
\hline \multicolumn{2}{|c|}{ Parameters } & \multicolumn{7}{|c|}{ Experimental periods } & \multirow[t]{2}{*}{ Overall } \\
\hline & & $1^{\text {st }}$ week & $2^{\text {nd }}$ week & $3^{\text {rd }}$ week & $4^{\text {th }}$ week & $5^{\text {th }}$ week & $6^{\text {th }}$ week & $7^{\text {th }}$ week & \\
\hline \multirow[t]{2}{*}{$\mathrm{RBC}$} & $\mathrm{Gl}$ & $\begin{array}{l}5.53 \pm \\
0.86^{\mathrm{aA}}\end{array}$ & $\begin{array}{c}6.02 \pm \\
0.85^{\text {abA }}\end{array}$ & $\begin{array}{l}5.52 \pm \\
0.88^{\mathrm{aA}}\end{array}$ & $\begin{array}{c}6.10 \pm \\
0.82^{\text {abA }}\end{array}$ & $\begin{array}{l}5.65 \pm \\
0.86^{\mathrm{aA}}\end{array}$ & $\begin{array}{l}6.41 \pm \\
0.85^{\mathrm{bA}}\end{array}$ & $\begin{array}{l}6.27 \pm \\
0.87^{\mathrm{bA}}\end{array}$ & $\begin{array}{c}5.92 \pm \\
0.71^{\mathrm{A}}\end{array}$ \\
\hline & GII & $\begin{array}{c}7.56 \pm \\
0.95^{\mathrm{abB}}\end{array}$ & $\begin{array}{l}7.38 \pm \\
0.96^{\mathrm{aB}}\end{array}$ & $\begin{array}{l}7.04 \pm \\
0.90^{\mathrm{aB}}\end{array}$ & $\begin{array}{l}7.15 \pm \\
0.70^{\mathrm{aB}}\end{array}$ & $\begin{array}{l}6.98 \pm \\
0.85^{\mathrm{aB}}\end{array}$ & $\begin{array}{l}8.23 \pm \\
0.86^{c B}\end{array}$ & $\begin{array}{c}8.13 \pm \\
0.84^{\text {bcB }}\end{array}$ & $7.48 \pm 0.82^{\mathrm{B}}$ \\
\hline \multirow[t]{2}{*}{$\mathrm{HB}$} & $\mathrm{Gl}$ & $\begin{array}{c}11.11 \pm \\
0.87^{\mathrm{aA}}\end{array}$ & $\begin{array}{l}11.42 \pm \\
0.90^{\mathrm{abA}}\end{array}$ & $\begin{array}{l}11.22 \pm \\
0.87^{\mathrm{bA}}\end{array}$ & $\begin{array}{l}11.12 \pm \\
1.10^{\mathrm{bA}}\end{array}$ & $\begin{array}{c}10.98 \pm \\
0.89^{\mathrm{aA}}\end{array}$ & $\begin{array}{c}11.97 \pm \\
0.91^{\mathrm{bA}}\end{array}$ & $\begin{array}{l}11.51 \pm \\
0.85^{\mathrm{abA}}\end{array}$ & $\begin{array}{c}11.42 \pm \\
0.68^{\mathrm{A}}\end{array}$ \\
\hline & GII & $\begin{array}{l}12.97 \pm \\
0.91^{\mathrm{bcB}}\end{array}$ & $\begin{array}{l}12.22 \pm \\
0.85^{\mathrm{aB}}\end{array}$ & $\begin{array}{l}12.48 \pm \\
0.82^{\mathrm{abB}}\end{array}$ & $\begin{array}{c}12.21 \pm \\
1.12^{\mathrm{aB}}\end{array}$ & $\begin{array}{l}11.94 \pm \\
0.97^{\mathrm{aB}}\end{array}$ & $\begin{array}{c}13.56 \pm \\
0.93^{\mathrm{CB}}\end{array}$ & $\begin{array}{l}13.17 \pm \\
0.86^{\text {bcB }}\end{array}$ & $\begin{array}{c}12.63 \pm \\
0.85^{\mathrm{B}}\end{array}$ \\
\hline \multirow[t]{2}{*}{ ESR } & $\mathrm{Gl}$ & $\begin{array}{c}4.79 \pm \\
0.68^{\mathrm{abA}}\end{array}$ & $\begin{array}{c}4.93 \pm \\
0.71^{\mathrm{abA}}\end{array}$ & $\begin{array}{c}4.82 \pm \\
0.73^{\mathrm{abA}}\end{array}$ & $\begin{array}{l}5.12 \pm \\
0.76^{\mathrm{abA}}\end{array}$ & $\begin{array}{l}4.64 \pm \\
0.71^{\mathrm{aA}}\end{array}$ & $\begin{array}{l}5.44 \pm \\
0.71^{\mathrm{AA}}\end{array}$ & $\begin{array}{c}5.12 \pm \\
0.74^{\mathrm{bcA}}\end{array}$ & $\begin{array}{c}4.96 \pm \\
1.60^{A}\end{array}$ \\
\hline & GII & $\begin{array}{l}5.56 \pm \\
0.70^{\mathrm{aB}}\end{array}$ & $\begin{array}{l}5.58 \pm \\
0.75^{\mathrm{aB}}\end{array}$ & $\begin{array}{l}5.42 \pm \\
0.66^{\mathrm{aB}}\end{array}$ & $\begin{array}{l}5.70 \pm \\
0.77^{\mathrm{aB}}\end{array}$ & $\begin{array}{l}5.31 \pm \\
0.68^{\mathrm{aB}}\end{array}$ & $\begin{array}{l}6.52 \pm \\
0.74^{\mathrm{B}}\end{array}$ & $\begin{array}{l}6.18 \pm \\
0.76^{\mathrm{BB}}\end{array}$ & $5.74 \pm 0.74^{\mathrm{B}}$ \\
\hline \multirow[t]{2}{*}{ PCV } & $\mathrm{Gl}$ & $\begin{array}{l}32.29 \pm \\
0.89^{\mathrm{abA}}\end{array}$ & $\begin{array}{l}32.58 \pm \\
1.20^{\mathrm{abA}}\end{array}$ & $\begin{array}{l}32.52 \pm \\
1.16^{\mathrm{abA}}\end{array}$ & $\begin{array}{c}32.94 \pm \\
1.14^{\mathrm{bA}}\end{array}$ & $\begin{array}{c}31.74 \pm \\
1.01^{\mathrm{aA}}\end{array}$ & $\begin{array}{c}32.94 \pm \\
0.98^{\mathrm{bA}}\end{array}$ & $\begin{array}{c}31.91 \pm \\
0.85^{\mathrm{aA}}\end{array}$ & $\begin{array}{c}32.42 \pm \\
0.77^{\mathrm{A}}\end{array}$ \\
\hline & GII & $\begin{array}{c}36.86 \pm \\
1.11^{\mathrm{cB}}\end{array}$ & $\begin{array}{l}36.29 \pm \\
1.22^{\mathrm{bcB}}\end{array}$ & $\begin{array}{c}37.03 \pm \\
1.18^{\mathrm{cB}}\end{array}$ & $\begin{array}{c}33.64 \pm \\
1.13^{\mathrm{aB}}\end{array}$ & $\begin{array}{l}35.42 \pm \\
0.86^{\mathrm{bB}}\end{array}$ & $\begin{array}{c}37.10 \pm \\
1.30^{\circ B}\end{array}$ & $\begin{array}{c}36.53 \pm \\
1.13^{\mathrm{CB}}\end{array}$ & $\begin{array}{c}36.11 \pm \\
1.21^{\mathrm{B}}\end{array}$ \\
\hline \multirow[t]{2}{*}{ MCV } & $\mathrm{Gl}$ & $\begin{array}{c}59.89 \pm \\
2.94^{\mathrm{bA}}\end{array}$ & $\begin{array}{l}55.49 \pm \\
2.74^{\text {abA }}\end{array}$ & $\begin{array}{l}60.72 \pm \\
2.12^{\mathrm{bA}}\end{array}$ & $\begin{array}{l}56.20 \pm \\
2.72^{\mathrm{abA}}\end{array}$ & $\begin{array}{l}57.69 \pm \\
2.81^{\text {abA }}\end{array}$ & $\begin{array}{c}52.50 \pm \\
2.44^{\mathrm{aA}}\end{array}$ & $\begin{array}{c}52.18 \pm \\
2.46^{\mathrm{aA}}\end{array}$ & $\begin{array}{c}56.45 \pm \\
1.93^{\mathrm{A}}\end{array}$ \\
\hline & GII & $\begin{array}{l}49.84 \pm \\
2.53^{\text {abcB }}\end{array}$ & $\begin{array}{l}50.15 \pm \\
2.42^{\text {abcB }}\end{array}$ & $\begin{array}{c}53.78 \pm \\
2.59^{\mathrm{CB}}\end{array}$ & $\begin{array}{l}47.77 \pm \\
1.93^{\mathrm{abB}}\end{array}$ & $\begin{array}{l}51.68 \pm \\
2.23^{\mathrm{bcB}}\end{array}$ & $\begin{array}{l}45.63 \pm \\
1.87^{\mathrm{aB}}\end{array}$ & $\begin{array}{c}46.21 \pm \\
1.75^{\mathrm{aB}}\end{array}$ & $\begin{array}{c}50.15 \pm \\
1.58^{\mathrm{B}}\end{array}$ \\
\hline \multirow[t]{2}{*}{$\mathrm{MCH}$} & $\mathrm{Gl}$ & $\begin{array}{c}20.46 \pm \\
1.89^{A}\end{array}$ & $\begin{array}{c}19.39 \pm \\
1.65^{\mathrm{A}}\end{array}$ & $\begin{array}{c}20.88 \pm \\
1.82^{\mathrm{A}}\end{array}$ & $\begin{array}{c}18.97 \pm \\
1.78^{\mathrm{A}}\end{array}$ & $\begin{array}{c}19.91 \pm \\
1.74^{\mathrm{A}}\end{array}$ & $\begin{array}{c}19.15 \pm \\
1.60^{\mathrm{A}}\end{array}$ & $\begin{array}{c}18.77 \pm \\
1.61^{\mathrm{A}}\end{array}$ & $\begin{array}{c}19.63 \pm \\
0.86^{\mathrm{A}}\end{array}$ \\
\hline & GII & $\begin{array}{c}17.54 \pm \\
1.64^{\mathrm{B}}\end{array}$ & $\begin{array}{c}16.84 \pm \\
1.37^{\mathrm{B}}\end{array}$ & $\begin{array}{c}18.10 \pm \\
1.58^{\mathrm{B}}\end{array}$ & $\begin{array}{c}17.30 \pm \\
1.33^{\mathrm{B}}\end{array}$ & $\begin{array}{c}17.43 \pm \\
1.56^{\mathrm{B}}\end{array}$ & $\begin{array}{c}16.71 \pm \\
1.40^{\mathrm{B}}\end{array}$ & $\begin{array}{c}16.53 \pm \\
1.41^{\mathrm{B}}\end{array}$ & $\begin{array}{c}17.21 \pm \\
0.83^{\mathrm{B}}\end{array}$ \\
\hline \multirow[t]{2}{*}{$\mathrm{MCHC}$} & $\mathrm{GI}$ & $\begin{array}{c}33.98 \pm \\
1.16^{\mathrm{aA}}\end{array}$ & $\begin{array}{l}34.97 \pm \\
1.37^{\mathrm{abcA}}\end{array}$ & $\begin{array}{c}34.42 \pm \\
1.21^{\mathrm{aA}}\end{array}$ & $\begin{array}{c}33.63 \pm \\
1.40^{\mathrm{aA}}\end{array}$ & $\begin{array}{l}34.51 \pm \\
1.47^{\mathrm{abA}}\end{array}$ & $\begin{array}{c}36.25 \pm \\
1.37^{c}\end{array}$ & $\begin{array}{c}35.98 \pm \\
1.37^{\mathrm{bc}}\end{array}$ & $\begin{array}{c}34.92 \pm \\
0.59\end{array}$ \\
\hline & GII & $\begin{array}{l}35.10 \pm \\
1.18^{\mathrm{abB}}\end{array}$ & $\begin{array}{c}33.62 \pm \\
1.37^{\mathrm{aB}}\end{array}$ & $\begin{array}{c}33.43 \pm \\
1.13^{\mathrm{aB}}\end{array}$ & $\begin{array}{c}36.16 \pm \\
1.47^{\mathrm{bB}}\end{array}$ & $\begin{array}{c}33.56 \pm \\
1.50^{\mathrm{aB}}\end{array}$ & $\begin{array}{c}36.55 \pm \\
1.43^{b}\end{array}$ & $\begin{array}{c}35.71 \pm \\
1.32^{\mathrm{b}}\end{array}$ & $\begin{array}{c}34.923 \pm \\
1.21\end{array}$ \\
\hline
\end{tabular}

RBC: Red blood cells $\left(\times 10^{6} / \mathrm{mm}^{3}\right)$; HB: Haemoglobin $\left(\mathrm{g} \mathrm{dl}^{-1}\right)$; ESR: Erythrocyte sedimentation rate $\left(\mathrm{mm} \mathrm{hr}^{-1}\right)$; PCV: Packed cell volume (\%), MCV: Mean corpuscular volume $\left(\mu^{3}\right)$; $\mathrm{MCH}$ : Mean corpuscular haemoglobin $(\mu \mu \mathrm{g})$; MCHC: Mean corpuscular haemoglobin concentration (\%); GI: Unstressed animal group; GII: Stressed animal group; Within rows means with different letters (a, b, c, d) differ significantly $(p<0.05)$; Within columns means with different letters (A, B) differ significantly $(p<0.05)$ between the stressed and unstressed for the particular parameter

investigation periods for physiological profiles like RT, RR, $\mathrm{PR}, \mathrm{HR}$ and ST and also among the investigation weeks, a considerable significant $(p<0.05)$ difference was reported. Similarly affected crossbred cows had suffered significantly higher $(p<0.05)$ than in unaffected cows.

Data analysis revealed that highly significant $(p<0.05)$ value on the physiological parameters (RT, RR, PR, HR and ST) were got in crossbred cows of ANI which were suffered with walking stress. It was also noted that the physiological parameter such as respiration rate was affected significantly especially immediately after hard draught work and pulse rate as well as the rectal temperature were also increased followed by RR in the cattle species. Furthermore it was revealed that RT and RR are the indicative stress markers in domestic livestock species to assess the magnitude and severity of stress (Daramola and Adeloye, 2009; Sejian et al., 2010b; Perumal et al., 2016). In caprine and bovine species were also observed similar kind of reports that rectal temperature and respiratory rate were increased as the animals were exercising or walking more severely (Kasa et al., 1995; Coulon and Pradel, 1997; Perumal et al., 2016). Based on the available literatures in the domestic animal species, it was clearly indicated that the bovine species 
Table 3: Correlation coefficients among the physiological and heamatological parameters of unstressed crossbred cows of Andaman and Nicobar Islands

\begin{tabular}{|c|c|c|c|c|c|c|c|c|c|c|c|c|}
\hline & RT & $\mathrm{RR}$ & PR & $\mathrm{HR}$ & ST & TRBC & $\mathrm{HB}$ & ESR & PCV & $\mathrm{MCV}$ & $\mathrm{MCH}$ & $\mathrm{MCHC}$ \\
\hline RT & 1 & $0.96^{* *}$ & $0.97^{* *}$ & 0.47 & $0.93^{* *}$ & $0.73^{*}$ & $0.94^{* *}$ & $0.82^{* *}$ & 0.25 & -0.63 & -0.36 & $0.95^{* *}$ \\
\hline $\mathrm{RR}$ & & 1 & $0.96^{* *}$ & 0.52 & $0.91^{* *}$ & $0.70^{*}$ & $0.91^{* *}$ & $0.74^{*}$ & 0.12 & $-0.76^{*}$ & -0.44 & $0.93^{* *}$ \\
\hline PR & & & 1 & 0.49 & $0.91^{* *}$ & 0.65 & $0.92^{* *}$ & $0.75^{*}$ & 0.14 & -0.61 & -0.35 & $0.92^{* *}$ \\
\hline $\mathrm{HR}$ & & & & 1 & 0.21 & 0.66 & 0.52 & 0.52 & -0.22 & $-0.78^{*}$ & -0.69 & 0.64 \\
\hline ST & & & & & 1 & 0.57 & $0.88^{*}$ & 0.65 & 0.19 & -0.51 & -0.32 & $0.84^{* *}$ \\
\hline TRBC & & & & & & 1 & $0.83^{*}$ & $0.87^{* *}$ & 0.34 & $-0.95^{* *}$ & $-0.92^{* *}$ & $0.79^{*}$ \\
\hline $\mathrm{HB}$ & & & & & & & 1 & $0.94^{* *}$ & 0.45 & $-0.74^{*}$ & -0.55 & $0.87^{* *}$ \\
\hline ESR & & & & & & & & 1 & 0.52 & $-0.79^{*}$ & -0.68 & $0.74^{*}$ \\
\hline PCV & & & & & & & & & 1 & -0.21 & -0.20 & -0.15 \\
\hline $\mathrm{MCV}$ & & & & & & & & & & 1 & $0.95^{* *}$ & $-0.77^{*}$ \\
\hline $\mathrm{MCH}$ & & & & & & & & & & & 1 & -0.52 \\
\hline $\mathrm{MCHC}$ & & & & & & & & & & & & 1 \\
\hline
\end{tabular}

RT: Rectal Temperature; RR: Respiration Rate; PR: Pulse Rate; HR: Heart Rate; ST: Skin Temperature; TRBC: Total Red Blood Cells; HB: Heamoglobin; ESR: Erythrocyte Sedimentation Rate; PCV: Packed Cell Volume; MCV: Mean Corpuscular Volume; $\mathrm{MCH}$ : Mean Corpuscular Heamoglobin; MCHC: Mean Corpuscular Heamoglobin Concentration; *: Correlation coefficients were significant, $p<0.05 ;{ }^{* *}$ : Correlation coefficients were highly significant, $p<0.01$

Table 4: Correlation coefficients among the physiological and heamatological parameters of stressed crossbred cows of Andaman and Nicobar Islands

\begin{tabular}{|c|c|c|c|c|c|c|c|c|c|c|c|c|}
\hline & $\mathrm{RT}$ & $\mathrm{RR}$ & PR & $\mathrm{HR}$ & ST & TRBC & $\mathrm{HB}$ & ESR & PCV & MCV & $\mathrm{MCH}$ & $\mathrm{MCHC}$ \\
\hline RT & 1 & $0.95^{* *}$ & $0.96^{* *}$ & $0.88^{* *}$ & $0.76^{* *}$ & $0.88^{* *}$ & $0.95^{* *}$ & $0.75^{*}$ & 0.45 & $-0.73^{*}$ & -0.40 & $0.75^{*}$ \\
\hline $\mathrm{RR}$ & & 1 & $0.97^{* *}$ & $0.93^{* *}$ & $0.74^{*}$ & $0.87^{*}$ & $0.93^{* *}$ & $0.77^{*}$ & 0.49 & $-0.82^{*}$ & -0.41 & $0.72^{*}$ \\
\hline PR & & & 1 & $0.89^{* *}$ & $0.86^{* *}$ & $0.85^{* *}$ & $0.95^{* *}$ & $0.81^{*}$ & 0.67 & -0.65 & -0.43 & 0.65 \\
\hline $\mathrm{HR}$ & & & & 1 & 0.65 & $0.91^{* *}$ & $0.86^{* *}$ & $0.78^{*}$ & 0.41 & $-0.78^{*}$ & -0.66 & 0.66 \\
\hline ST & & & & & 1 & $0.78^{*}$ & $0.88^{* *}$ & 0.64 & $0.94^{* *}$ & -0.32 & 0.30 & 0.32 \\
\hline TRBC & & & & & & 1 & $0.90^{* *}$ & $0.91^{* *}$ & 0.50 & -0.68 & $-0.78^{*}$ & 0.56 \\
\hline $\mathrm{HB}$ & & & & & & & 1 & $0.89^{* *}$ & 0.62 & -0.70 & -0.45 & 0.62 \\
\hline ESR & & & & & & & & 1 & 0.33 & $-0.79^{*}$ & $-0.71^{*}$ & $0.86^{*}$ \\
\hline PCV & & & & & & & & & 1 & 0.26 & -0.32 & -0.21 \\
\hline MCV & & & & & & & & & & 1 & 0.50 & $-0.94^{* *}$ \\
\hline $\mathrm{MCH}$ & & & & & & & & & & & 1 & -0.58 \\
\hline $\mathrm{MCHC}$ & & & & & & & & & & & & 1 \\
\hline
\end{tabular}

*: Correlation coefficients were significant, $p<0.05 ;{ }^{* *}$ : Correlation coefficients were highly significant, $p<0.01$

was severely affected with severe stress that undergone walking and/or grazing or exercising as reflected from the measurement of different antioxidant, physiological and heamatological profiles. It was reported that the physiological parameters were significantly increased in the present study due to effect of walking stress than the values were observed in the earlier studies for bovine species under the island ecosystem of ANI (Sunder et al., 2016). Measurement of respiration rate indirectly understand to assess the heat production effect during the stress condition (Berhan et al., 2006) and higher RR in crossbred cows may have more homeostatic relevance for the excessive heat dissipation and lower/optimum body temperature maintenance (Rahardja et al., 2011). Significantly higher respiratory rate was reported in the stressed animal as it is important for the thermoregulation of the body systems (Sejian et al., 2012; Perumal et al., 2016). Further, it was also noted that the investigation was carried out during dry hot season causes increased the detrimental 
effects due to walking stress or exercise leads to higher heat load and ultimately increased the RR in crossbred cows. Stress due to walking and exercising has also increased severe energy imbalance and/or deficit of energy because the locomotor activity of cows spends substantial amount of energy for the purpose of walking and/or exercise. Moreover, amount of energy was also decreased when the respiratory muscular activity was increased due to significantly greater respiratory movements especially during stress condition to disseminate or dissipate the heat from the body during the process of walking or exercise.

Pulse rate of animals is a suggestive marker to the circulation homeostasis primarily along with the other metabolic profiles of the animals (Sejian et al., 2010a). Pulse rate in group II cows has increased significantly when compared to the control group of crossbred cows after exposure to walking stress during the dry season in the present study. The present study data was clearly suggested that there are significant correlations between metabolic heat production and heart rate (Marai et al., 2007). Similarly, the ST was also higher significantly in the walking thermo stressed group of animals than unstressed control group of animals, however, it was lesser than the RT of the same crossbred cows as because higher sweating inturn reduce the ST. Measurement of sweating was also used to assess the heat stress response in domestic livestock species like bovine, ovine and equine (Kumar et al., 2011). The present study fact clearly indicated that besides the heat has been relieved through respiratory evaporative cooling, the animal also needs to eliminate heat from the body through cutaneous evaporation indicated the severity of stresses on these animals in physiological parameters. Moreover, it was also indicated that RT acts as a suggestive suitable marker of walking stress in the ovine species (Sejian et al., 2010a). Similar types of result were obtained in bovine species i.e. in Hariana bullocks (Yadav and Dhaka, 2001), Surungi bullocks (Behera et al., 2008) and mithun (Perumal et al., 2016). In Indian subcontinent, Tomar and Joshi (2008) was in Kenkatha bullock, Shelke and Siddiqui (2009) was in Red Kandhari bullocks, Atakare and Siddiqui (2009) was in Deoni bullocks, Singh and Nanavati (2013) was in crossbred bullocks and Perumal et al. (2016) was in mithun reported similar findings as in the present study.

\subsection{Heamatological profiles}

Significant $(p<0.05)$ difference was noticed between the affected and unaffected crossbred cows for the heamatological profiles like TRBC, HB, ESR, PCV, MCV, $\mathrm{MCH}$ and $\mathrm{MCHC}$. However, these heamatological attributes were significantly differed $(p<0.05)$ among the various experimental weeks. Stress affected group (II) has significantly $(p<0.05)$ higher TRBC, HB, ESR, PCV and MCHC than unaffected group I whereas $\mathrm{MCV}$ and $\mathrm{MCH}$ were lower significantly $(p<0.05)$ in group II.

Severe metabolic, haematological and acid-base \& ionic changes in animals were observed which are exposed to severe and hard exercise or walk or work load and are depended on the magnitude or severity of the cumulative effects of physiological and physical trauma related to the stressful events and which are specie-specific (Hassanein, 2010). Physical stress causes acidosis leads to disturbances in haematological and antioxidant profiles (Moyes et al., 2006). Serum profiles in bovine provide clear indicators to assess the status of internal environment and understanding the causes of impairment in homeostasis as evidenced by marked alterations in physiogical indexes under various internal as well as external environmental conditions (Sattar and Mirza, 2009). Composition of blood clearly demonstrated the abnormal pathological and normal physiological states of the animals inducing by stress (Satue et al., 2009).

Blood profiles like PCV, RBC, MCV, MCHC and $\mathrm{MCH}$ are used to assess the acclimatization of livestock to various adverse environmental situations (Koubkova et al., 2002). Significant adverse effect on blood constituents were observed in stressed crossbred cows as clearly suggested that these affected animals were severely suffered with severe haemoconcentration. In the walk stressed cows, the blood indices were significantly higher than that the values were reported in normal unaffected cattle. Significantly higher blood indices in the stressed animal were due to adaptive/ acclimatization mechanism shown by the crossbred cows in the ANI in the present study. Oxygen consumption rate is increased in general as whenever the animal is walking or running or exercising. In ANI, the crossbred cows were managed under the semi-intensive rearing system, walking long distance to search the fodder or feed in the jungle especially during dry season/months. Therefore these animals need increased concentration of the oxygen to achieve higher muscular activity which inturn increased RBC production and concentration of $\mathrm{Hb}$ in crossbred cows. Further, in the current study, the cows were not allowed to take water or feed or fodder while walking inturn caused haemoconcentration leads to increased the value of blood indices especially RBC, HB, PCV and ESR (Sejian et al., 2012; Perumal et al., 2016). Similar kind of findings was also reported in other domestic animal species (Garcia-Belenguer et al., 1996: cattle; Sejian et al., 2012: sheep; Perumal et al., 2016: mithun). Severe dehydration was reported during thermal stress in livestock species in general prospective where increased level of $\mathrm{Hb}$ and PCV was noted (Marai et al., 2007; McManus et al., 2009). Severe water deprivation could have aggravated during walking stress in these crossbred cows. Moreover, the present experiment was conducted in dry heat season causes severe deleterious effects on these blood/heamatological attributes. Observed that walking during dry months is a highly energy consuming activity for the crossbred cows and causes higher sweating during the walking process inturn increased haemoconcentration. Moreover, $\mathrm{Hb}$ and PCV are to be the suitable indices of the organic response to the exercising or 
walking stress (Sejian et al., 2010b; Perumal et al., 2016).

\subsection{Antioxidant profiles}

Significant $(p<0.05)$ difference was noticed between the treated and untreated control animals for the antioxidant profiles like TAC, CAT \& GSH: Figure 1 and SOD: Figure 2).

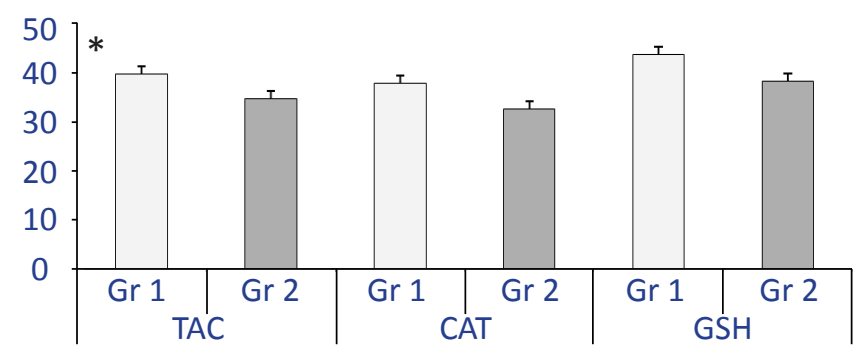

Figure 1: Effect of walking stress on antioxidants activity in crossbred cows of Andaman and Nicobar Islands (* indicates

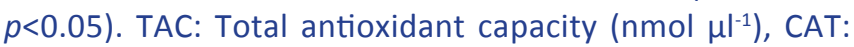
Catalase (nmol $\left.\left.\mathrm{min}^{-1}\right|^{-1}\right)$ and GSH: Glutathione $\left(\mathrm{nmol}^{-1}\right)$. Gr 1 : Unstressed animal group, Gr 2: Stressed animal group.

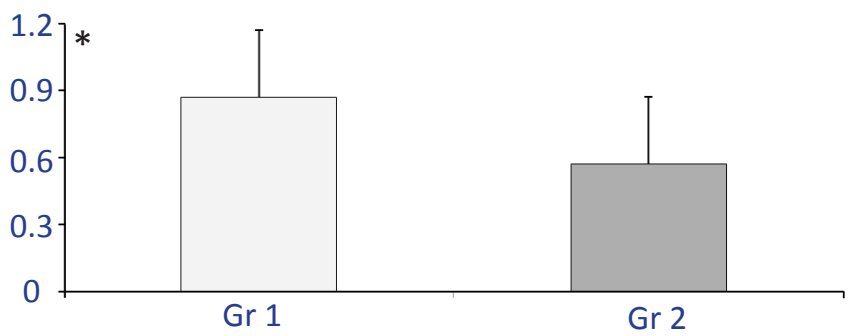

Figure 2: Effect of walking stress on superoxide dismutase activity in crossbred cows of Andaman and Nicobar Islands (* indicates $p<0.05)$. Gr 1: Unstressed animal group; Gr 2: Stressed animal group

In walk stressed cows, the level of the antioxidants was reduced significantly $(p<0.05)$ than in unstressed control crossbred cow group. Similar to other parameters, lipid peroxide profiles like malondialdehyde (MDA: Figure 3) level was increased significantly $(p<0.05)$ in the walk stress affected crossbred cows than in unaffected stress free crossbred cows.

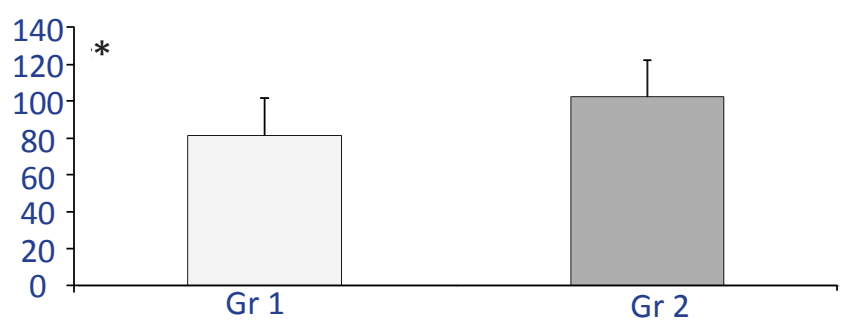

Figure 3: Effect of walking stress on malondialdehyde (MDA) production in crossbred cows of Andaman and Nicobar Islands (* indicates $p<0.05$ ). Gr 1: Unstressed animal group, Gr 2: Stressed animal group

Significantly $(p<0.05)$ reduced concentration of antioxidant profiles (GSH, SOD, CAT and TAC) was observed in the walk stressed crossbred cow groups and significantly $(p<0.05)$ higher concentration of oxidative profile (MDA) was noted in walk and heat stressed crossbred cows (Figure 1) in the present study. Different varieties of stresses cause deficiency of concentration of antioxidants because the neutralization or prevent production of lipid peroxide by the antioxidants. Different free radical oxidations are stimulated in animal species by different types of stressors and the final products of lipid peroxidation accumulate in different system (Yarovan, 2008). Lipid peroxide concentration was increased significantly $(p<0.05)$ in treatment cows in the present study as same kind of report was observed that heat stress and /or walking stress induce(s) excessive production of lipid peroxides (Bernabucchi et al., 2002; Sivakumar et al., 2010; Perumal et al., 2016). It was also proposed that exercise/ walking stress has to generate free radicals/lipid peroxides easily by the fallowing mechanisms (1) higher production of the of catecholamines like epinephrine, nor-epinephrine and other catecholamines causes increased production of lipid peroxides whenever they are inactivated metabolically, (2) increased production of lactic acid which can produce a strongly damaging one (hydroxyl) from a weakly damaging free radical (superoxide) and (3) secondary muscle damage leads to inflammatory responses with overexertion (Sen, 1995). Walking and/or thermal stresses can be controlled or counteracted with proper and higher supplementation of suitable antioxidants which are very useful to the livestock species (Sejian et al., 2012) as these antioxidant systems are essential compounds and/or systems which delay/minimise the self autoxidation through inhibition of the free radicals formation or by interfering the free radical propagation by different various mechanisms (Brewer, 2011). Through this, the antioxidant system helps to neutralize the free radical to protect the cells from damage during the stressful condition any.

\subsection{Correlation study}

Present study of stress effects on physiological, heamatological attributes revealed a positive correlation with significant $(p<0.05)$ was observed between the RT and RR, PR, ST, TRBC, HB, ESR \& MCHC. Similarly positive correlation with significant $(p<0.05)$ was observed between the respiratory rate and PR, ST, TRBC, HB, ESR \& MCHC and significant $(p<0.05)$ negative correlation was observed with MCV. Similarly pulse rate has positively correlated with significant $(p<0.05)$ with $\mathrm{HB}, \mathrm{ESR}, \mathrm{MCHC}$ and ST. And also positive correlation with significant $(p<0.05)$ was noticed between TRBC and $H B, E S R$ \& MCHC and significantly correlated negatively with $\mathrm{MCV}$ and $\mathrm{MCH}$. Same correlation pattern was observed in control unstressed (Table 3) and walk stressed (Table 4) animal groups.

\section{Conclusion}

The crossbred cows requires a suitable grazing/walking and feeding policy or strategy to prevent or minimise the deleterious effect stimulated by walking and dry season to 
the cows of ANI. However, detailed systematic proper study need to be carried out during both dry and monsoon seasons in different age groups of cow. And an ideal methodology need to form for supplementation of suitable feed, electrolytes \& antioxidants to minimise the stresses.

\section{Acknowledgement}

Authors acknowledge the supports from the Director, ICARCentral Island Agricultural Research Institute, Port Blair, Andaman and Nicobar Islands and Head of the Animal Science Division of the institute.

\section{References}

Atakare, S.P., Siddiqui, M.F., 2009. Evaluation of draught ability traits of Deoni cattle. M.V.Sc. Thesis, Maharashtra Animal and Fishery Science University, Nagpur.

Behera, B.K., Mohapatra, A.K., Behera, D., Swain, S., 2008. Effect of draft and season on physiological responses of bullocks. Journal of Agricultural Engineering 45(2), April - June, 2008. http://epubs.icar.org.in/ejournal/ index.php/JAE/article/view/14550.

Berhan, T., Puchala, R., Goetsch, A.L., Merkel, R.C., 2006. Effects of walking speed and forage consumption on energy expenditure and heart rate by Alpine does. Small Ruminant Research 63(1-2), 119-124.

Bernabucchi, V., Ronchi, B., Lacetera, N., Nardone, A., 2002. Markers of oxidative status in plasma and erythrocytes of transition dairy cows during hot season. Journal of Dairy Science 85, 2173.

Brewer, M.S., 2011. Natural antioxidants: sources, compounds, mechanisms of action, and potential applications. Comprehensive Review in Food Science and Food Safety 10, 221-247.

Coulon, J.B., Pradel, P., 1997. Effect of walking on roughage intake and milk yield and composition of Montbeliarde and Tarentaise dairy cows. Ann Zootech 46, 139-146.

D’Hour, P., Hauwuy, A., Coulon, J.B., Garel, J.P., 1994. Walking and dairy cattle performance. Annales de Zootechnie 43, 369-378.

Daramola, J.O., Adeloye, A.A., 2009. Physiological adaptation to humid tropics with special references to the West African Dwarf (WAD) goat. Tropical Animal Health and Production 41, 1005-1016.

Garcia-Belenguer, S., Palacio, J., Gascon, M., Acena, C., Revilla, R., Mormede, P., 1996. Differences in the biological stress responses of two cattle breeds to walking up to mountain pastures in the Pyrenees. Veterinary Research 7, 515-526.

Gustafson, G.M., Luthman, J., Burstedt, E., 1993. Effect of daily exercise on performance, feed efficiency and energy balance of tied dairy cows. Acta Agriculturae Scandinavica 43, 219-227.

Hassanein, L.H., 2010. The physiological and physical response to capture stress in sharks. Plymouth Stud Science 4, 413-422.

Kasa, I.W., Hill, M.K., Thwaites, C.J., Baillie, N.D., 1995.
Physiological effects of exercise in male and female Saanen goats at the same body weight but different feed intake. Small Ruminant Research 16, 83-86.

Koubkova, M., Knizkova, I., Kunc, P., Hartlova, H., Flusser, J., Dolezal, O., 2002. Influence of high environmental temperature and evaporative cooling on some physiological, haematological and biochemical parameters in high-yielding dairy cows. Czech Journal of Animal Science 47, 309-318.

Kumar, B.V.S., Kumar, A., Kataria, M., 2011. Effect of heat stress in tropical livestock and different strategies for its amelioration. Journal of Stress Physiology and Biochemistry 7, 45-54.

Livestock Census of India (17 $\left.{ }^{\text {th }}\right)$, 2003. All India Report. Ministry of Agriculture Department of Animal Husbandry, Dairying and Fisheries, Krishi Bhawan, New Delhi, India, 2007.

Livestock Census of India (18 $\left.{ }^{\text {th }}\right)$, 2007. All India Report. Ministry of Agriculture Department of Animal Husbandry, Dairying and Fisheries, Krishi Bhawan, New Delhi, India, 2007.

Livestock Census of India (19 $\left.{ }^{\text {th }}\right)$, 2012. All India Report. Ministry of Agriculture Department of Animal Husbandry, Dairying and Fisheries, Krishi Bhawan, New Delhi, India, 2012.

Manteca, X., Smith, A.J., 1994. Effects of poor forage conditions on the behaviour of grazing ruminants. Tropical Animal Health and Production 26, 129-138.

Marai, I.F.M., El-Darawany, A.A., Fadiel, A., Abdel-Hafez, M.A.M., 2007. Physiological traits as affected by heat stress in sheep - a review. Small Ruminant Research 71, 1-12.

McManus, C., Paluda, G.R., Louvandini, H., Gugel, R., Sasaki, L.C.B., Paiva, S.R., 2009. Heat tolerance in Brazilian sheep: physiological and blood parameters. Tropical Animal Health and Production 41, 95-101.

Moyes, C.D., Fragoso, N., Musy, M.K., Brill, R.W., 2006. Predicting post release survival in large pelagic fish. Transaction of American Fisheries Society 135, 13891397.

Perumal, P., Khan, M.H., Chang, S., Sangma, C.T.R., 2016. Effect of walking and summer stress on physiological, heamatological and antioxidant profiles in mithun bulls. Indian Journal of Animal Sciences 86(7), 751-757.

Rahardja, D.P., Toleng, A.L., Lestari, V.S., 2011. Thermoregulation and water balance in fat-tailed sheep and Kacang goat under sunlight exposure and water restriction in a hot and dry area. Animal 5(10), 1587-1593.

Sattar, A., Mirza, R.H., 2009. Haematological parameters in exotic cows during gestation and lactation under subtropical conditions. Pakistan Veterinary Journal 29, 129-132.

Satue, K., Blanco, O., Munoz, A., 2009. Age-related differences 
in the haematological profile of Andalusian broodmares of Carthusian strain. Veterinary Medicine-Czech 54, 175-182.

Sejian, V., Maurya, V.P., Naqvi, S.M.K., 2010a. Effect of thermal, nutritional and combined (thermal and nutritional) stresses on growth and reproductive performance of Malpura ewes under semi-arid tropical environment. Journal of Animal Physiology and Animal Nutrition 95, 252-258.

Sejian, V., Maurya, V.P., Naqvi, S.M.K., 2010b. Physiological adaptability and growth performance of Malpura ewes subjected to thermal and nutritional stress under semiarid tropical environment. Tropical Animal Health and Production 42, 1763-1770.

Sejian, V., Singh, A.K., Sahoo, A., Naqvi, S.M.K., 2012. Effect of mineral mixture and antioxidant supplementation on growth, reproductive performance and adaptive capability of Malpura ewes subjected to heat stress. Journal of Animal Physiology and Animal Nutrition 98, 72-83.

Sen, C.K., 1995. Oxidants and antioxidants in exercise. Journal of Applied Physiology 79, 675-686.

Shelke, H.S., Siddiqui, M.F., 2009. Evaluation of draught ability traits of Red Kandhari cattle. M.V.Sc. thesis Maharashtra Animal and Fishery Science University, Nagpur.
Singh, B., Nanavati, S., 2013. Study on the physiological and haematological changes during the work performance of crossbred bullocks. Haryana Veterinarian 52, 88-89.

Sivakumar, A.V.N., Singh, G., Varshney, V.P., 2010: Antioxidants supplementation on acid base balance during heat stress in goats. Asian-Australian Journal of Animal Sciences 23, 1462-1468.

Sunder, J., Kundu, A., Kundu, M.S., 2016. Scientific goat farming in Andaman \& Nicobar Islands. Technical Report. https://www.researchgate.net/publication/299468622

Tomar, S.S., Joshi, S.K., 2008. Characterization of Kenkatha cattle (Famous draught breed of Bundelkhand). J.N.K.V.V. Technical Bull. DRS/2008/01.

Vision 2050 of ICAR-CIARI., 2011. Published by ICAR-Central Island Agricultural Research Institute, Port Blair, Andaman and Nicobar Islands.

Yadav, A.S., Dhaka, S.S., 2001. Efficiency of sustained work and its influence on physiological responses in young bulls of Hariana cattle. Asian-Australian Journal of Animal Science 14(8), 1062-1066.

Yarovan, N.I., 2008. Effect of zeolites on adaptation processes in cows. Russian Agricultural Science 34, 120-122. 